\title{
A LEMMA ON NEARBY CYCLES AND ITS APPLICATION TO THE TAME LUBIN-TATE SPACE
}

\author{
JEAN-FranÇOIS DAT
}

\begin{abstract}
This note is concerned with a cohomological consequence of a geometric construction due to Yoshida, which relates the tame level of the Lubin-Tate tower to some Deligne-Lusztig variety of Coxeter type. More precisely, we show that the equivariant morphism in cohomology which follows from Yoshida's construction is an isomorphism, whatever the coefficients are. In particular, this gives a conceptual explanation to the observation that $\ell$-adic cohomologies indeed were "the same", once computed independently on each side (by Boyer, resp. Lusztig). This also gives a "simple" proof of the absence of torsion in the integral cohomology of the tame Lubin-Tate space. Our main tool is a general result on vanishing cycles for schemes with semi-stable reduction which generalizes previous results of Zheng and Illusie. In rough terms, this states that the restriction of the nearby cycles complex to a closed stratum is the push-forward of its restriction to the corresponding open stratum.
\end{abstract}

\section{Main result}

Let $K$ be a non-archimedean local field with ring of integers $\mathcal{O}$, uniformizer $\varpi$ and residue field $\mathbb{F}_{q}$.

1.1. The tame Lubin-Tate space. We fix an integer $d$ and a formal $\mathcal{O}$-module $\mathbb{H}_{d}$ of dimension 1 and height $d$ over $\overline{\mathbb{F}}_{q}$. We refer the reader e.g., to [10] for the definition of such an object and related structures. Let us denote by $\breve{\mathcal{O}}$ the completion of the maximal unramified extension of $\mathcal{O}$. We consider the $\breve{\mathcal{O}}$-formal scheme $\widehat{\mathcal{M}_{n}}$ which parameterizes deformations of $\mathbb{H}_{d}$ together with an "order $n$ Drinfeld level structure". It is the formal spectrum of a complete local $\breve{\mathcal{O}}$-algebra $A_{n}$. The algebra $A_{0}$ is formally smooth over $\breve{\mathcal{O}}$ of relative dimension $d-1$, and $A_{n}$ is finite over $A_{0}$ with deck transformation group $\mathrm{GL}_{d}\left(\mathcal{O} / \varpi^{n}\right)$. Let us denote by $\mathcal{M}_{n}$ the generic fiber of $\widehat{\mathcal{M}}_{n}$, an analytic space over the non-archimedean field $\breve{K}:=\breve{\mathcal{O}}[1 / p]$. So $\mathcal{M}_{0}$ is an open ball of dimension $d-1$ and $\mathcal{M}_{n}$ is a Galois étale covering of $\mathcal{M}_{0}$ with group $\mathrm{GL}_{d}\left(\mathcal{O} / \varpi^{n}\right)$. In this note, we will focus on $\mathcal{M}_{1}$ with its action by $\mathrm{GL}_{d}\left(\mathbb{F}_{q}\right)$.

1.2. A Deligne-Lusztig variety. Let $w=(1,2, \ldots, d) \in \mathfrak{S}_{d}$ be the Coxeter element of the Weyl group of $\mathrm{GL}_{d}$. We denote by $\mathcal{X}(w)$ the variety over $\overline{\mathbb{F}}_{q}$ which parameterizes Borel subgroups of $\mathrm{GL}_{d, \mathbb{F}_{q}}$ which are in relative position $w$ with their image under the Frobenius map relative to $\mathbb{F}_{q}$. It carries an action of $\mathrm{GL}_{d}\left(\mathbb{F}_{q}\right)$. Further we denote by $\pi: \widetilde{\mathcal{X}}(w) \longrightarrow \mathcal{X}(w)$ the $\mathrm{GL}_{d}\left(\mathbb{F}_{q}\right)$-equivariant $\mathbb{F}_{q^{d}}^{\times}$-torsor defined in $[5$, p. 111]. These objects admit the following more concrete description, see $[5$,

Received by the editors June 24, 2011. 
p. 117]. First, $\mathcal{X}(w)$ identifies with the open subset $\Omega_{\mathbb{F}_{q}}^{d-1}$ of $\mathbb{P}_{\mathbb{F}_{q}}^{d-1}$ defined, in projective coordinates, by the non-vanishing of the $\operatorname{determinant} \operatorname{det}\left(\left(x_{i}^{q^{j}}\right)_{0 \leqslant i, j \leqslant d-1}\right)$. In other words, $\Omega_{\mathbb{F}_{q}}^{d-1}$ is the complement of $\mathbb{F}_{q}$-rational hyperplanes, the so-called Drinfeld upper half-space. Second, $\widetilde{\mathcal{X}}(w)$ identifies with the open subset of $\mathbb{A}_{\mathbb{F}_{q}}^{d}$ defined by the equation $\operatorname{det}\left(\left(x_{i}^{q^{j}}\right)_{0 \leqslant i, j \leqslant d-1}\right)^{q-1}=(-1)^{d-1}$.

1.3. A relation between these objects. Let $\breve{K}_{d}$ denote the ramified extension of $\breve{K}$ generated by a $q^{d}-1$-root of $\varpi$. In [11], Yoshida constructed a $\mathrm{GL}_{d}\left(\mathbb{F}_{q}\right)$-equivariant integral model of $\mathcal{M}_{1} \hat{\otimes}_{\breve{K}} \breve{K}_{d}$, whose special fiber contains an open subset $\mathrm{GL}_{d}\left(\mathbb{F}_{q}\right)$ isomorphic to $\widetilde{\mathcal{X}}(w)$, and which is smooth at points of this open subset. Therefore, the general formalism of nearby/vanishing cycles provides a morphism in $D^{b}\left(\Lambda \mathrm{GL}_{d}\left(\mathbb{F}_{q}\right)\right)$ :

$$
R \Gamma\left(\mathcal{M}_{1}^{\mathrm{ac}}, \Lambda\right) \longrightarrow R \Gamma(\tilde{\mathcal{X}}(w), \Lambda) .
$$

Here $^{\text {ac }}$ denotes a base change to an algebraic closure of $\breve{K}$ and $\Lambda$ is $\mathbb{Z} / \ell^{n} \mathbb{Z}$ or $\mathbb{Z}_{\ell}$.

However, the cohomological tools at hand only allowed him to relate the cuspidal part of the $\overline{\mathbb{Q}}_{\ell}$-cohomologies of both objects, as well as the alternating sum. This is kind of frustrating, because the explicit computations of Boyer [4], resp. Lusztig [7], for $\Lambda=\overline{\mathbb{Q}}_{\ell}$ suggest a stronger relation, at least for $\ell$-adic coefficients.

Our aim here is to improve on the cohomological tools, in order to prove:

Theorem 1.1. The morphism (1.1) is an isomorphism. Moreover, by duality we also get an isomorphism

$$
R \Gamma_{\mathrm{c}}\left(\mathcal{M}_{1}^{\mathrm{ac}}, \Lambda\right) \stackrel{\sim}{\longrightarrow} R \Gamma_{\mathrm{c}}(\widetilde{\mathcal{X}}(w), \Lambda) .
$$

The cohomological tool we provide is a property of nearby cycles for semi-stable schemes and is the object of Section 2, see Theorem 2.1. The application to the tame Lubin-Tate space is done in Section 3.

After completing this note, the author has learned from Illusie that a statement very close to, but slightly weaker than Theorem 2.1 already appears in [12, Lemma 5.6] (and generalizes a previous particular case of $[6,1.5]$ ). However, our enhanced version is necessary for our application to the Lubin-Tate space and probably for further similar applications such as to Drinfeld spaces.

Here is an example of a consequence of the foregoing theorem. We will use it in a forthcoming paper on a realization of the mod- $\ell$ local Langlands correspondence.

Corollary 1.1. The $\mathbb{Z}_{\ell}$-modules $H_{\mathrm{c}}^{i}\left(\mathcal{M}_{1}, \mathbb{Z}_{\ell}\right)$ are torsion-free.

Indeed, the torsion-freeness in the case of $\widetilde{\mathcal{X}}(w)$ follows from Lemma 3.9 and Corollary 4.3 of $[3]^{1}$. We note that Boyer has announced torsion-freeness results for the whole Lubin-Tate tower, but the proof here is arguably easier.

1.4. An open question. The action of $\mathrm{GL}_{d}(\mathcal{O}) /\left(1+\varpi \mathcal{M}_{d}(\mathcal{O})\right)$ on the cohomology groups $H^{i}\left(\mathcal{M}_{1}, \Lambda\right)$ is the restriction of an action by "cohomological correspondences" of the Hecke algebra $\mathcal{H}_{\Lambda}\left(\mathrm{GL}_{d}(K),\left(1+\varpi \mathcal{M}_{d}(\mathcal{O})\right)\right)$ of compactly supported measures on $\mathrm{GL}_{d}(K)$ which are bi-invariant by the compact open subgroup $1+\varpi \mathcal{M}_{d}(\mathcal{O})$. An interesting problem would be to determine this action.

\footnotetext{
${ }^{1}$ I thank Olivier Dudas for supplying this reference to me
} 


\section{Nearby cycles}

2.1. The semi-stable setting. Let $S=\operatorname{Spec}(W)$ be a strict henselian trait with generic point $\eta$ and closed point $s$ of residue characteristic $p$. We choose a geometric point $\bar{\eta}$ over $\eta$ and denote by $\Delta$ the corresponding Galois (inertia) group. Let $\Lambda$ be either $\mathbb{Z}_{\ell} / \ell^{k} \mathbb{Z}_{\ell}$ or $\mathbb{Z}_{\ell}$. For an $S$-scheme $X$, we denote by $R \Psi: D^{+}\left(X_{\eta}, \Lambda\right) \longrightarrow$ $D^{+}\left(X_{s}, \Lambda \Delta\right)$ the (absolute) nearby cycles functor ${ }^{2}$.

We will assume that $X$ has "generalized" semi-stable reduction, i.e., that it is flat over $S$, with smooth generic fiber $X_{\eta}$, and with special fiber $X_{s}$ a "tame" normal crossings divisor. The latter condition means that each closed point of $X_{s}$ has an étale neighborhood which admits an étale map to some scheme of the form $\operatorname{Spec}\left(W\left[X_{1}, \ldots, X_{n}\right] /\left(X_{1}^{m_{1}} \cdots X_{n}^{m_{n}}-\varpi\right)\right)$ where $\varpi$ is a uniformizer of $W$ and the $m_{i}$ are prime to $p$.

Write $X_{s}=\sum_{i \in I} e_{i} Y_{i}$ as a sum of irreducible reduced divisors with multiplicities. For $\emptyset \neq J \subseteq I$, we put

- $Y_{J}:=\bigcap_{i \in J} Y_{i}$ and $i_{J}: Y_{J} \hookrightarrow X$ its closed immersion.

- $Y_{J}^{0}:=$ smooth part of $\left(Y_{J} \backslash \bigcup_{i \notin J} Y_{i}\right)$ and $j_{J}: Y_{J}^{0} \hookrightarrow X$ the corresponding locally closed immersion.

We extend the notation to $J=\emptyset$ by putting $Y_{\emptyset}:=X$, so that $Y_{\emptyset}^{0}=X_{\eta}$. For $J \subseteq K$ we have $Y_{K} \subseteq Y_{J}$, and if $Y_{K}$ is not the empty scheme, then it has pure codimension $\operatorname{codim}\left(Y_{K}, Y_{J}\right)=|K \backslash J|$.

Theorem 2.1. For $\emptyset \neq J \subseteq I$, the canonical morphism

$$
i_{J, *} i_{J}^{*}(R \Psi(\Lambda)) \longrightarrow R j_{J, *} j_{J}^{*}(R \Psi(\Lambda))
$$

is an isomorphism.

Remark 2.1. The main differences with Zheng's result in [12, Lemma 5.6] is that we allow generalized semi-stable models and deeper strata. Also our proof is different, and it is not clear how to get deeper strata with Zheng's approach.

Proof. Note that this statement is equivalent to the following one :

(*) For any closed point $x \in Y_{J} \backslash Y_{J}^{0}$, we have $i_{x}^{!} i_{J}^{*}(R \Psi(\Lambda))=0$.

Therefore it is local in the étale topology, and we may assume that

$$
X=\operatorname{Spec}\left(W\left[x_{1}, \ldots, x_{n}\right] /\left(x_{1}^{e_{1}} \cdots x_{n}^{e_{n}}-\varpi\right)\right) .
$$

More precisely, all we have to do is to prove statement $(*)$ for $x$ the point with ideal $\left(\varpi, x_{1}, \ldots, x_{n}\right)$ in $X$ as above. Further, by invariance of nearby cycles under smooth morphisms, we may assume that all $e_{i}$ are non-zero. In this case, $I=\{1, \ldots, n\}$ and $Y_{J}$ is non-empty, smooth and irreducible for all $J \subseteq I$. In particular $Y_{J}^{0}=Y_{J} \backslash \bigcup_{i \notin J} Y_{i}$. Note also that $Y_{I}=\{x\}=Y_{I}^{0}$, so our statement is non-empty only for $J \neq I$. In fine we are left to prove that for our particular $X$ above, the following holds:

$$
\text { for any } J \varsubsetneqq I \text { we have, } i_{I}^{!} i_{J}^{*}(R \Psi(\Lambda)=0 \text {. }
$$

Step 1. Our next task is to reduce further the latter statement to the case where $e_{i}=1$ for all $i \in I$. To do this, we will follow the idea of a similar reduction step in

\footnotetext{
${ }^{2}$ Sometimes also called "vanishing" cycles functor
} 
the proof of [9, Proposition 6]. Let $e$ be a common multiple of the $e_{i}$ 's which is prime to $p$, and put

$$
\widetilde{X}:=\operatorname{Spec}\left(W\left[y_{1}, \ldots, y_{n}\right] /\left(y_{1}^{e} \cdots y_{n}^{e}-\varpi\right)\right) .
$$

The map $x_{i} \mapsto y_{i}^{e / e_{i}}$ defines a flat covering $\widetilde{X} \stackrel{f}{\longrightarrow} X$ with deck transformation group $H=\prod_{i} \mu_{e / e_{i}}$ (roots of unity) acting by $\left(\zeta_{1}, \ldots, \zeta_{n}\right) \cdot\left(y_{1}, \ldots, y_{n}\right)=\left(\zeta_{1} y_{1}, \ldots, \zeta_{n} y_{n}\right)$ and which is Galois étale over $\eta$. Therefore, the sheaf $f_{\eta, *}\left(\Lambda_{\widetilde{X}_{\eta}}\right)$ on $X$ is a sheaf of $\Lambda[H]$-modules and the adjunction map $\Lambda_{X_{\eta}} \longrightarrow f_{\eta, *}\left(\Lambda_{\widetilde{X}_{\eta}}\right)$ is an isomorphism $\Lambda_{X_{\eta}} \stackrel{\sim}{\longrightarrow}$ $f_{\eta, *}\left(\Lambda_{\tilde{X}_{\eta}}\right)^{H}$. Note that taking $H$-invariants is a finite projective limit, hence commutes with pull-back functors. On the other hand, the derived $H$-invariant functor $R_{H}$ on complexes of $\Lambda[H]$-sheaves coincides with the functor $R \operatorname{Hom}_{\Lambda[H]}(\Lambda,-)$, which commutes with direct images and extraordinary inverse images (see SGA 4, Exp. XVIII, Corollary 3.1.12.2 for the latter). Therefore we get an isomorphism

$$
i_{I}^{!} i_{J}^{*} R \Psi(\Lambda) \stackrel{\sim}{\longrightarrow} R_{H}\left(i_{I}^{!} i_{J}^{*} R \Psi\left(f_{\eta, *} \Lambda\right)\right) .
$$

Now, we have a canonical isomorphism $R \Psi\left(f_{\eta, *} \Lambda\right) \stackrel{\sim}{\longrightarrow} R f_{s, *}(R \Psi(\widetilde{X}, \Lambda))$ since $f$ is finite, and this induces in turn an isomorphism

$$
i_{I}^{!} i_{J}^{*} R \Psi\left(f_{\eta, *} \Lambda\right) \stackrel{\sim}{\longrightarrow} R f_{\mathrm{s}, *}\left(\widetilde{i}_{I}^{!} \widetilde{i}_{J}^{*} R \Psi(\widetilde{X}, \Lambda)\right),
$$

in which $\widetilde{i}_{I}^{*}$ and $\widetilde{i}_{J}^{*}$ are analogous to $i_{I}^{*}$ and $i_{J}^{*}$ but relative to $\widetilde{X}$. Finally, let $\widetilde{W}:=$ $W[t] /\left(t^{e}-\varpi\right)$, a strict henselian local extension of $W$ with uniformizer $t$ and inertia group $\widetilde{\Delta}$. We may see $\widetilde{X}$ as a $\widetilde{W}$-scheme by sending $t$ to $y_{1} \cdots y_{n}$ and thus we get a nearby cycles complex $R \widetilde{\Psi}(\widetilde{X}, \Lambda) \in D^{+}\left(\widetilde{X}_{s}, \Lambda \widetilde{\Delta}\right)$ relative to $\widetilde{W}$, and related to the previous one by the formula

$$
R \Psi(\widetilde{X}, \Lambda) \simeq \operatorname{Ind}_{\widetilde{\Delta}}^{\Delta}(R \widetilde{\Psi}(\widetilde{X}, \Lambda)) .
$$

On the other hand, as a $\widetilde{W}$-scheme, $\widetilde{X}$ is equal to $\widetilde{X}\left[y_{1}, \ldots, y_{n}\right] /\left(y_{1} \cdots y_{n}-t\right)$. Therefore, the three displayed isomorphisms above reduce our statement to the case where all multiplicities are 1.

Step 2. We now assume all multiplicities are 1 and will reduce the vanishing of $i_{I}^{!} i_{J}^{*}(R \Psi(\Lambda))$ to its analog for rational nearby cycles. More precisely, let us put $i: X_{s} \hookrightarrow X$ and $j=j_{\emptyset}: X_{\eta} \hookrightarrow X$, and let us assume that for $J \varsubsetneqq I$ we have

$$
i_{I}^{!} i_{J}^{*}\left(i^{*} R j_{*}(\Lambda)\right)=0 .
$$

Note that, by construction, $i^{*} R j_{*}(\Lambda) \stackrel{\sim}{\longrightarrow} R_{\Delta} R \Psi(\Lambda)$, where $R_{\Delta}$ is the derived functor of the $\Delta$-invariant functor. Now, recall the following results from [8] (only the second one requires the multiplicity 1 assumption):

(1) The action of $\Delta$ on $R \Psi(\Lambda)$ factors through the tame quotient $\Delta_{t}$, [8, Satz $2.23]$.

(2) The action of $\Delta$ on each $R^{p} \Psi(\Lambda)$ is trivial, [8, Korollar 2.25] or SGA 7, I, Theorem 3.3.

Let $T$ be a progenerator of the tame inertia group $\Delta_{t}$. As a consequence of i) and [8, Satz 1.1] we have a distinguished triangle

$$
R_{\Delta} R \Psi(\Lambda) \longrightarrow R \Psi(\Lambda) \stackrel{T-1}{\longrightarrow} R \Psi(\Lambda) \stackrel{+1}{\longrightarrow},
$$


and as a consequence of ii), the endomorphism $T-1$ of $R \Psi(\Lambda)$ is nilpotent. Then, applying $i_{I}^{!} i_{J}^{*}$ to the above triangle yields, assuming (2.2), a triangle

$$
0 \longrightarrow i_{I}^{!} i_{J}^{*} R \Psi(\Lambda) \stackrel{T-1}{\longrightarrow} i_{I}^{!} i_{J}^{*} R \Psi(\Lambda) \stackrel{+1}{\longrightarrow},
$$

which means that $T-1$ is an automorphism of $i_{I}^{!} i_{J}^{*} R \Psi(\Lambda)$. Since it is also nilpotent, we get $i_{I}^{!} i_{J}^{*} R \Psi(\Lambda)=0$.

Step 3: Proof of (2.2). In fact it is more convenient to prove the dual (and equivalent) statement that $i_{I}^{*} i_{J}^{!} j_{!}(\Lambda)=0$. Let us start with the simplicial resolution:

$$
j_{!}(\Lambda) \stackrel{\sim}{\longrightarrow}\left(\Lambda \longrightarrow \bigoplus_{i \in I} i_{i, *}(\Lambda) \longrightarrow \cdots \longrightarrow \bigoplus_{K \subset I,|K|=p} i_{K, *}(\Lambda) \longrightarrow \cdots \longrightarrow i_{I, *}(\Lambda)\right) \text {. }
$$

For each subset $K \subseteq I$, we have $Y_{J} \cap Y_{K}=Y_{J \cup K}$, which is smooth of codimension $|J \backslash K|$ in $Y_{K}$. Therefore by purity, we have an isomorphism

$$
i_{J}^{!} i_{K, *}(\Lambda) \stackrel{\sim}{\longrightarrow} i_{J}^{*} i_{K, *}(\Lambda)[-2|J \backslash K|]
$$

and thus an isomorphism

$$
i_{I}^{*} i_{J}^{!} i_{K, *}(\Lambda) \stackrel{\sim}{\longrightarrow} \Lambda[-2|J \backslash K|] .
$$

The first page of the spectral sequence attached to the above resolution then reads:

$$
E_{1}^{p, q}=\left(\bigoplus_{K \subseteq I,|K|=p, q=2|J \backslash K|} \Lambda\right) \Rightarrow R^{p+q}\left(i_{I}^{*} i_{J}^{!} j_{!}(\Lambda)\right) .
$$

More precisely, the line $E_{1}^{\bullet, q}$ is zero unless $q$ is even. In this case, put $k_{2}:=|J|-\frac{1}{2} q$. Then $E_{1}^{\bullet, q}$ decomposes as a sum of complexes $E_{1}^{\bullet, q}=\sum_{K_{2} \subseteq J,\left|K_{2}\right|=k_{2}} \mathcal{C}_{K_{2}}^{\bullet}$, where

$$
\mathcal{C}_{K_{2}}^{\bullet}=\left(\Lambda \longrightarrow \cdots \longrightarrow \bigoplus_{K_{1} \subseteq I \backslash J,\left|K_{1}\right|=p-k_{2}}^{\longrightarrow} \Lambda \longrightarrow \cdots \longrightarrow \Lambda\right)
$$

which is, up to a shift by $-k_{2}$, the chain complex associated to the simplicial power set of $I \backslash J$. Since $I \backslash J \neq \emptyset$, this chain complex is acyclic. Therefore $E_{2}^{p q}=0$ for all $p, q$, and finally $i_{I}^{*} i_{J} j_{!}(\Lambda)=0$, as desired.

2.2. Reinterpretation in terms of analytic tubes and formal schemes. We continue with the setting of 2.1 but we assume further that $W$ is $\varpi$-adically complete and write $\mathcal{K}:=W[1 / p]$ for its field of fractions. Let $X_{\eta}^{\text {an }}$ denote the $\mathcal{K}$-analytic space (in the sense of Berkovich) attached to $X_{\eta}$ via the GAGA functor. There is a specialization map $\left|X_{\eta}^{\mathrm{an}}\right| \stackrel{\mathrm{sp}}{\longrightarrow}\left|X_{s}\right|$ between the underlying topological spaces. The inverse image $\mathrm{sp}^{-1}(Z)$ of a locally closed subset $Z \subset\left|X_{s}\right|$ is locally closed in the Berkovich topology and inherits a canonical analytic structure. It is called the analytic tube of $Z$ in $X_{\eta}^{\text {an }}$. Berkovich has defined the étale site of such an analytic space in [1]. According to [2, Corollary 3.5], the cohomology of the tube is related to $Z$ by a canonical isomorphism in $D^{b}(\Lambda \Delta)$

$$
R \Gamma\left(\mathrm{sp}^{-1}(Z)^{\mathrm{ac}}, \Lambda\right) \stackrel{\sim}{\longrightarrow} R \Gamma(Z, R \Psi(X, \Lambda)),
$$

where $^{\text {ac }}$ denotes a base change to an algebraic closure of $\mathcal{K}$. Theorem 2.1 then implies the following statement. 
Corollary 2.1. With the notation of 2.1, the canonical restriction morphism

$$
R \Gamma\left(\mathrm{sp}^{-1}\left(Y_{J}\right)^{\mathrm{ac}}, \Lambda\right) \longrightarrow R \Gamma\left(\mathrm{sp}^{-1}\left(Y_{J}^{0}\right)^{\mathrm{ac}}, \Lambda\right)
$$

is an isomorphism.

For the application we have in mind, it is convenient to recast this in terms of formal schemes. Take up the notation above the Corollary and let $\widehat{X}_{Z}$ denote the formal completion of $X$ along $Z$. This belongs to a class of formal schemes $\mathfrak{X}$ over $W$ for which Berkovich has defined in [2] the notion of "analytic generic fiber" $\mathfrak{X}_{\eta}$, together with a nearby cycles functor ${ }^{3} R \Psi_{\eta}: D^{+}\left(\mathfrak{X}_{\eta}, \Lambda\right) \longrightarrow D^{+}\left(\mathfrak{X}_{s}, \Lambda\right)$ which computes the cohomology of $\mathfrak{X}_{\eta}$ via the usual formula

$$
R \Gamma\left(\mathfrak{X}_{\eta}^{\mathrm{ac}}, \mathcal{F}\right) \stackrel{\sim}{\longrightarrow} R \Gamma\left(\mathfrak{X}_{s}, R \Psi_{\eta}(\mathfrak{X}, \mathcal{F})\right) .
$$

In the case $\mathfrak{X}=\widehat{X}_{Z}$, we have $\mathfrak{X}_{s}=Z$ and $\mathfrak{X}_{\eta} \simeq \operatorname{sp}^{-1}(Z)$, and the main theorem of [2] exhibits a canonical isomorphism $R \Psi_{\eta}(\mathfrak{X}, \Lambda) \stackrel{\sim}{\longrightarrow} R \Psi(X, \Lambda)_{\mid Z}$ which explains isomorphism (2.3).

Corollary 2.2. With the notation of 2.1, there is a canonical isomorphism

$$
R \Gamma\left(\left(\widehat{X_{Y_{J}}}\right)_{\eta}^{\mathrm{ac}}, \Lambda\right) \stackrel{\sim}{\longrightarrow} R \Gamma\left(Y_{J}^{0}, R \Psi_{\eta}\left(\widehat{X_{Y_{J}}}, \Lambda\right)\right)
$$

\section{Application to the "tame" Lubin-Tate deformation space}

Here, we take up the notation of the introduction. Our former $W$ now becomes $\breve{\mathcal{O}}$.

3.1. Some notation. We focus on $\widehat{\mathcal{M}_{1}}=\operatorname{Spf}\left(A_{1}\right)$ and recall some of the constructions of Yoshida's in [11]. Note that the latter rather works with the spectrum $X:=\operatorname{Spec}\left(A_{1}\right)$. We will stick to his notation by writing $\widehat{X}:=\operatorname{Spf}\left(A_{1}\right)$. In $[11$, Section 4], a "semi-stable" $\mathrm{GL}_{d}\left(\mathbb{F}_{q}\right)$-equivariant modification of $X$ is constructed. It is a $\breve{\mathcal{O}}$-scheme $Z_{\text {st }}$, equipped with an action of $\mathrm{GL}_{d}\left(\mathbb{F}_{q}\right)$, endowed with an equivariant proper morphism $Z_{\text {st }} \stackrel{f}{\longrightarrow} X$ which induces an isomorphism of generic fibers, and whose completed local rings at points of the special fiber are of the usual "semistable" form, up to replacing polynomials by formal series. The closed fiber of $Z_{\mathrm{st}}$ over $X$ (meaning the fiber over the closed point of $X$ ), denoted by $Y_{\mathbb{P}, \text { st }}$ in loc. cit., is equivariantly isomorphic to a projective space $\mathbb{P}_{\overline{\mathbb{F}}_{q}}^{d-1}$ blown up at all $\mathbb{F}_{q}$-rational linear subspaces. As a component of the special fiber, it has multiplicity $q^{d}-1$ and meets the other components along the exceptional locus of this blow up. In other words, the open complement in $Y_{\mathbb{P}, \text { st }}$ of the other irreducible components, denoted by $Y_{\mathbb{P}, \text { st }}^{0}$, identifies with the Drinfeld upper half space $\Omega_{\mathbb{F}_{q}}^{d-1}$ (the complement in $\mathbb{P}_{\overline{\mathbb{F}}_{q}}^{d-1}$ of all $\mathbb{F}_{q}$-rational hyperplanes).

Corollary 3.1. Let us denote by $\widehat{Z}_{\mathrm{st}}$ the completion of $Z_{\mathrm{st}}$ along its closed fiber $Y_{\mathbb{P}, \mathrm{st}}$. There is a $\mathrm{GL}_{d}\left(\mathbb{F}_{q}\right)$-equivariant isomorphism

$$
R \Gamma\left(\mathcal{M}_{1}^{\mathrm{ac}}, \Lambda\right) \stackrel{\sim}{\longrightarrow} R \Gamma\left(\Omega_{\mathbb{F}_{q}}^{d-1}, R \Psi_{\eta}\left(\widehat{Z}_{\mathrm{st}}, \Lambda\right)\right)
$$

\footnotetext{
3 called "vanishing cycles" in loc. cit.
} 
Moreover, the complex $R \Psi_{\eta}\left(\widehat{Z}_{\mathrm{st}}, \Lambda\right)_{\mid \Omega_{\mathbb{F}_{q}}^{d-1}}$ is concentrated in cohomological degree 0 and $R^{0} \Psi_{\eta}\left(\widehat{Z}_{\mathrm{st}}, \Lambda\right)_{\mid \Omega_{\mathbb{F}_{q}}^{d-1}}$ is a locally constant sheaf in free $\Lambda$-modules of rank $q^{d}-1$.

Proof. In section 4.2 of [11], it is recalled how $\widehat{X}$ can be naturally identified to the completion $\widehat{\mathrm{Sh}_{\bar{s}}}$ of some Shimura variety Sh defined over $\mathcal{O}$ at some "supersingular" geometric point $\bar{s}$ of its special fiber. Then Yoshida exhibits a sequence of blow up's resulting in a (generalized) semi-stable model $\mathrm{Sh}_{\mathrm{st}} \stackrel{f}{\longrightarrow}$ Sh of the Shimura variety over $\mathcal{O}$. The relation with the local construction above is that $\widehat{Z}_{\text {st }}$ identifies with the formal completion $\widehat{\mathrm{Sh}}_{\mathrm{st}, f^{-1}(\bar{s})}$ of $\mathrm{Sh}_{\mathrm{st}}$ at the closed subscheme $f^{-1}(\bar{s})$ of its special fiber. In particular, $f^{-1}(\bar{s}) \simeq Y_{\mathbb{P}, \mathrm{st}}$. Therefore, the claimed isomorphism follows from Corollary 2.2, and the remaining statements follow from [9, Proposition 6].

Remark 3.1. In [11, Proposition 6.14 i)], Yoshida uses a result of Saito's to get an analog of the above statement in the Grothendieck group of $\Lambda \mathrm{GL}_{d}\left(\mathbb{F}_{q}\right)$-modules (and involving compactly supported cohomology on the open stratum).

3.2. Proof of Theorem 1.1. Now the task of describing the sheaf $R^{0} \Psi_{\eta}\left(\widehat{Z}_{\mathrm{st}}, \Lambda\right)_{\mid Y_{\mathbb{P}, \mathrm{st}}^{0}}$ was already achieved by Yoshida. Recall that $\Omega_{\mathbb{F}_{q}}^{d-1}$ is naturally equivariantly isomorphic to the Deligne-Lusztig base variety $\mathcal{X}(w)$ described in the introduction. Recall also the covering map $\pi: \widetilde{\mathcal{X}}(w) \longrightarrow \mathcal{X}(w)$.

Fact 1 (Yoshida). There is a $\mathrm{GL}_{d}\left(\mathbb{F}_{q}\right)$-equivariant isomorphism

$$
R^{0} \Psi_{\eta}\left(\widehat{Z}_{\mathrm{st}}, \Lambda\right)_{\mid \Omega_{\mathrm{F}_{q}}^{d-1}} \simeq \pi_{*}(\Lambda)
$$

Proof. We summarize Yoshida's argument for the convenience of the reader. The scheme $Z_{\text {st }}$ is obtained by a sequence of blow up's. The first stage simply consists in blowing up the closed point of $X$, see $[11,3.4]$. Denote by $Z_{1} \stackrel{f_{1}}{\longrightarrow} X$ this blow up. Its closed fiber, denoted by $Y_{\mathbb{P}}$, is a projective space $\mathbb{P}_{\overline{\mathbb{F}}_{q}}^{d-1}$. It is also the unique proper irreducible component of $Z_{1, s}$, it has multiplicity $q^{d}-1$, and it meets the other irreducible components at $\mathbb{F}_{q}$-rational hyperplanes. Then $Z_{\text {st }}$ is obtained by blowing up $Z_{1}$ at all rational subspaces of $Y_{\mathbb{P}}$. We thus have a factorization $f: Z_{\mathrm{st}} \stackrel{f^{1}}{\longrightarrow}$ $Z_{1} \stackrel{f_{1}}{\longrightarrow} X$ with $f^{1}$ a proper morphism which is an isomorphism above the tube of $\Omega_{\mathbb{F}_{q}}^{d-1}$. Therefore $f_{s, *}^{1}$ induces an isomorphism

$$
R \Psi_{\eta}\left(\widehat{Z}_{\mathrm{st}}, \Lambda\right)_{\mid \Omega_{\mathbb{F}_{q}}^{d-1}} \stackrel{\sim}{\longrightarrow} R \Psi_{\eta}\left(\widehat{Z}_{1}, \Lambda\right)_{\mid \Omega_{\mathbb{F}_{q}}^{d-1}}
$$

with $\widehat{Z}_{1}$ denoting the completion of $Z_{1}$ along its closed special fiber. Now, recall the extension $\breve{\mathcal{O}}_{d}:=\breve{\mathcal{O}}\left[\varpi^{1 /\left(q^{d}-1\right)}\right]$. If one forgets about the action of inertia, we have by definition an equality

$$
R \Psi_{\eta}\left(\widehat{Z}_{1}, \Lambda\right)=R \Psi_{\eta}\left(\widehat{Z}_{1} \times_{\breve{\mathcal{O}}} \breve{\mathcal{O}}_{d}, \Lambda\right)
$$

Consider now the normalization morphism $Z_{d} \stackrel{f}{\longrightarrow} Z_{1} \times_{\breve{\mathcal{O}}} \breve{\mathcal{O}}_{d}$. Since normalization is a local process which takes the algebra $\breve{\mathcal{O}}_{d}[X] /\left(X^{q^{d}-1}-\varpi\right)$ to $\breve{\mathcal{O}}_{d}^{q^{d}-1}$, the unique proper irreducible component of $Z_{d}$ has now multiplicity one, and the restriction of 
$f$ to the inverse image $U_{d}:=f^{-1}\left(\Omega_{\overline{\mathbb{F}}_{q}}^{d-1}\right)$ of $\Omega_{\overline{\mathbb{F}}_{q}}^{d-1}$ is étale of degree $q^{d}-1$. Since $f$ is a finite morphism, we thus get

$$
R^{0} \Psi_{\eta}\left(\widehat{Z}_{1}, \Lambda\right)_{\mid \Omega_{\mathbb{F}_{q}}^{d-1}} \simeq f_{*}\left(\Lambda_{U_{d}}\right)
$$

The computation of the normalization $U_{d}$ together with its action by $\mathrm{GL}_{d}\left(\mathbb{F}_{q}\right)$ is done in Section 5 of [11]. According to Proposition 6.15 of loc. cit., the covering $f: U_{d} \longrightarrow \Omega_{\mathbb{F}_{q}}^{d-1}$ is equivariantly isomorphic to the Deligne-Lusztig covering $\pi$.

Together with the previous corollary, this proves that the morphism (1.1) of the introduction is an isomorphism. Since $\mathcal{X}(w)$ is a smooth variety, and $\mathcal{M}_{1}$ is a smooth analytic space, we may apply Poincaré duality to get the isomorphism for compactly supported cohomology, as claimed in the main theorem.

Remark 3.2. The tube of $\Omega_{\mathbb{F}_{q}}^{d-1}$ in $\mathcal{M}_{1}$ is an affinoid subset of this non-quasi-compact analytic space, which was explicitly described by Weinstein in the case when $K$ has characteristic $p$. Corollary 2.1 shows that it captures all the cohomology of $\mathcal{M}_{1}$, in the sense that the restriction morphism

$$
R \Gamma\left(\mathcal{M}_{1}^{\mathrm{ac}}, \Lambda\right) \longrightarrow R \Gamma\left(\mathrm{sp}^{-1}\left(\Omega_{\mathbb{F}_{q}}^{d-1}\right)^{\mathrm{ac}}, \Lambda\right)
$$

is an isomorphism. In his ongoing work, Weinstein describes some remarkable affinoid subsets of deeper level Lubin-Tate spaces, which are attached to some BushnellKutzko data. One may hope that the techniques of this paper can be used to show that these affinoid subsets capture the part of the cohomology of the Lubin-Tate tower attached to these Bushnell-Kutzko data.

\section{Acknowledgments}

The author thanks J. Weinstein for a couple of motivating discussions around these topics. During the elaboration of this work, he was supported by the Institut Universitaire de France and by the Agence Nationale pour la Recherche under grant ANR-10-BLANC 0114.

\section{References}

[1] V.G. Berkovich, Étale cohomology for non-archimedean analytic spaces, Publ. Math. Inst. Hautes Études Sci. 78 (1993), 1-159.

[2] V.G. Berkovich, Vanishing cycles for formal schemes II, Invent. Math. 125 (1996), 367-390.

[3] C. Bonnafé and R. Rouquier, Coxeter orbits and modular representations, Nagoya Math. J. 183 (2006), 1-34.

[4] P. Boyer, Monodromie du faisceau pervers des cycles évanescents de quelques variétés de Shimura simples, Invent. Math. 177(2) (2009), 239-280.

[5] P. Deligne and G. Lusztig, Representations of reductive groups over finite fields, Ann. of Math. (2), 103(1) (1976), 103-161.

[6] L. Illusie, Sur la formule de Picard-Lefschetz, in 'Algebraic geometry 2000', Azumino (Hotaka), Adv. Stud. Pure Math. 36, 249-268. Math. Soc. Japan, Tokyo, 2002.

[7] G. Lusztig, Coxeter orbits and eigenspaces of Frobenius, Invent. Math. 38(2) (1976/77), 101-159.

[8] M. Rapoport and T. Zink, Über die lokale Zetafunktion von Shimuravarietäten, Monodromiefiltration und verschwindende Zyklen in ungleicher Characteristik, Invent. Math. 68 (1982), 21-101.

[9] T. Saito, $\epsilon$-factor of a tamely ramified sheaf on a variety, Invent. Math. 113(2) (1993), 389-417. 
[10] M. Strauch, Deformation spaces of one-dimensional formal modules and their cohomology, Adv. Math. 217(3) (2008), 889-951.

[11] T. Yoshida, On non-abelian Lubin-Tate theory via vanishing cycles, in 'Algebraic and arithmetic structures of moduli spaces (Sapporo 2007)', Adv. Stud. Pure Math. 58, 361-402, Math. Soc. Japan, Tokyo, 2010.

[12] W. Zheng, Sur la cohomologie des faisceaux l-adiques entiers sur les corps locaux, Bull. Soc. Math. France 136(3) (2008), 465-503.

Université Pierre et Marie Curie, Institut de Mathématiques de Jussieu, 4, Place Jussieu, 75005 PARIs, France

E-mail address: dat@math.jussieu.fr 
